\title{
Free amino acid content of the vitreous humour in cot deaths
}

\author{
W J A PATRICK* AND R W LOGAN $\dagger$ \\ Departments of *Perinatal and Paediatric Pathology and $\dagger$ Biochemistry, Royal Hospital for Sick Children, \\ Yorkhill, Glasgow
}

SUMMARY The concentration of 27 amino acids in the vitreous humour was analysed in 120 cot deaths. Baseline data for age and postmortem interval are presented and the concentrations compared with the normal reference range for plasma. The results do not indicate that a specific aminoacidopathy is a factor in cot deaths.

Since 1979 cot deaths have been investigated as part of an overall survey of postperinatal infant mortality in Glasgow and latterly (1981-2) in a national study for the whole of Scotland. 'In an attempt to exclude a specific aminoacidopathy as a cause of cot death the free amino acid content of the vitreous humour was analysed in a number of the Glasgow cases. To our knowledge this has not been previously documented, although there are two reports of vitreous amino acid studies in adults. ${ }^{2.3}$

\section{Methods}

Vitreous humour was aspirated before postmortem examination at the lateral angle of the eye from infants in an unselected series of 120 cot deaths in the Glasgow area (1979-84) representing $54 \%$ of the total number of cases in that period. The series included 72 boys and 48 girls and reflected the 3:2 male preponderance of cot deaths in our area and in other series. ${ }^{4}$

The cases were all infants under 1 year of age who had died suddenly and unexpectedly at home without a preceding illness or significant symptoms and as such all did not fit the standard definition of sudden infant death syndrome (SIDS). ${ }^{5}$

The postmortem examinations were carried out in accordance with the instructions of the Procurator Fiscal and followed a standard protocol which included histology of the major organs and selective microbiology. A specific cause of death was established in 13 cases $(11 \%)$ and nine had other incidental conditions.

One $\mathrm{ml}$ of vitreous humour was deproteinised by adding $50 \mathrm{mg}$ of salicylsulphonic acid and mixing the resultant solution. After centrifugation at $2000 \mathrm{~g}$ for 10 minutes the protein free supernatant was stored at $-25^{\circ} \mathrm{C}$ before analysis. $100 \mu \mathrm{l}$ samples were applied to both columns of a Technicon TSM aminoacid analyser with norleucine being used as an internal standard. Peak heights and widths were fed into a Wang 2200 computer for production of printed results expressed in $\mu \mathrm{mol} / \mathrm{l}$.

The results for 27 individual amino acids were analysed by normal distribution statistics, linear regression analysis, and the Mann-Whitney rank sum test for samples of unequal size by means of a

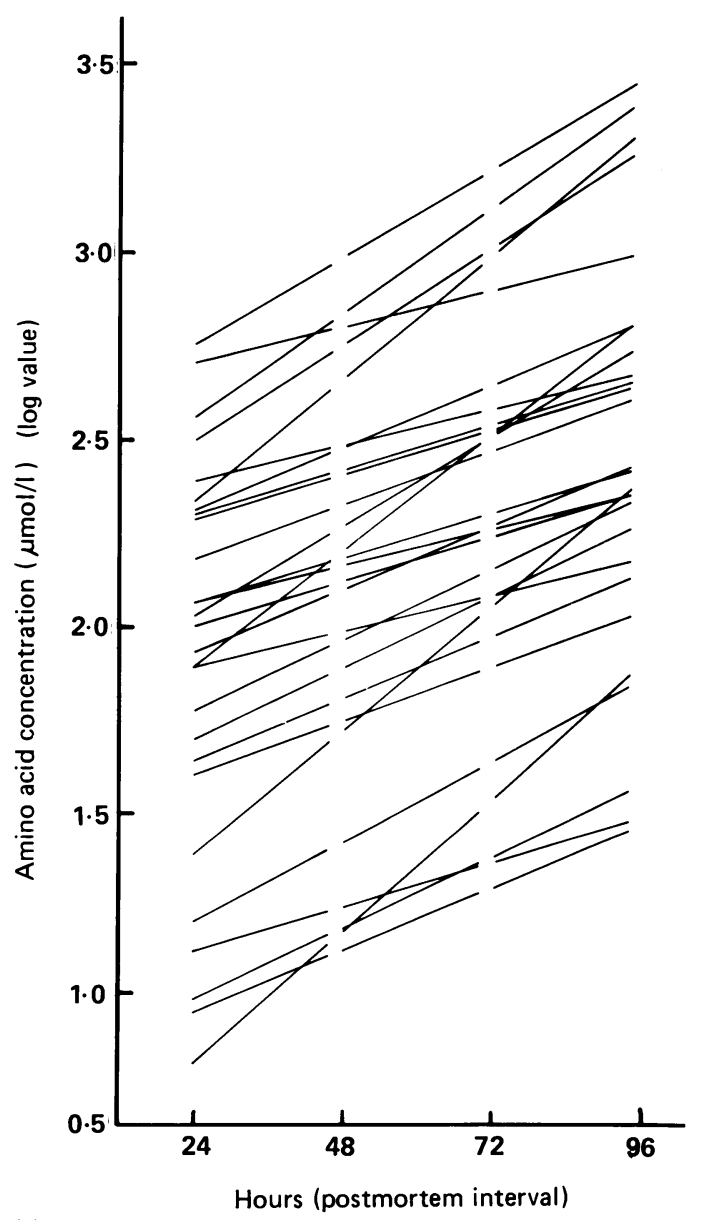

Fig 1 Logarithmic linear regression analysis of amino acid concentrations by postmortem interval. 
computer programme kindly made available by the department of surgery, Glasgow Royal Infirmary.

The cases were arbitrarily divided into those sampled $1-12$ hours $(n=32), 13-24$ hours $(n=26)$, $25-48$ hours $(n=35)$, and $49->96$ hours $(n=27)$ after the infant was last seen alive (postmortem interval), and by chronological age groups of $1-4$ weeks $(n=7), 5-13$ weeks $(n=45), 14-26$ weeks $(n=37)$, and $27-52$ weeks $(n=31)$.

\section{Results}

Logarithmic linear regression analysis showed that amino acid concentrations were directly related to

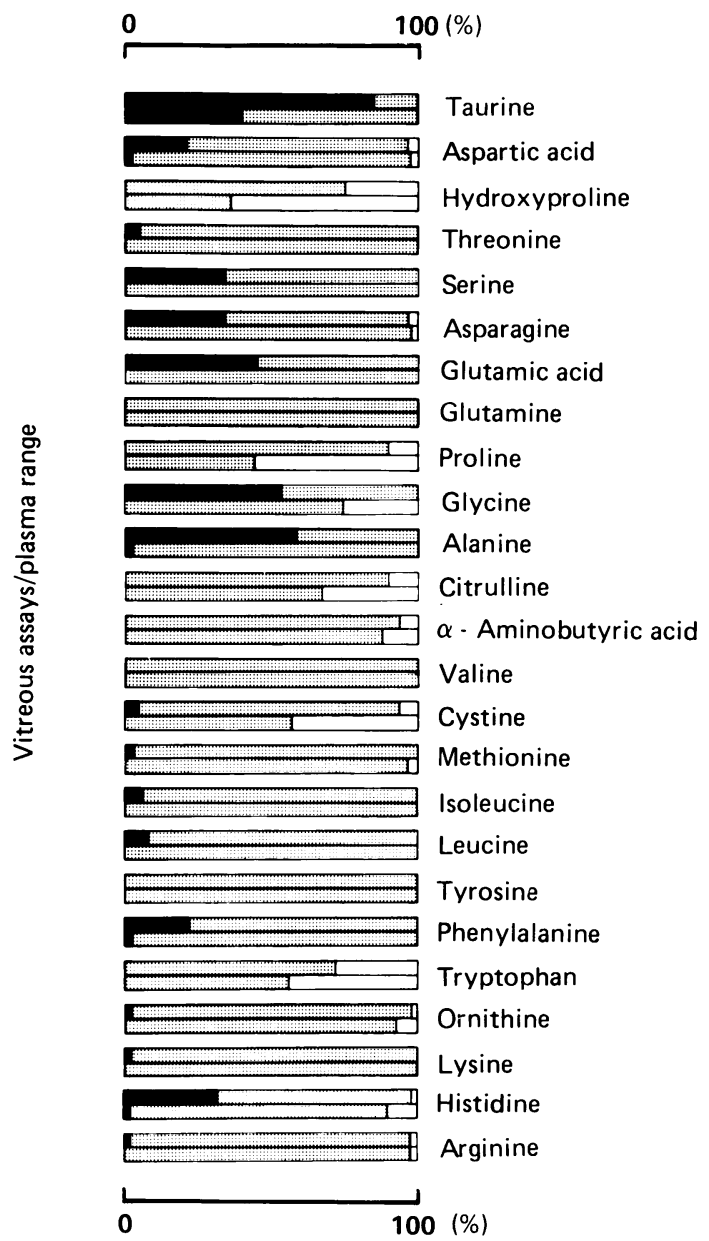

Fig 2 Percentage of vitreous amino acid assays above (black) within (shaded) and below (white) the normal plasma range in over 24 hour (upper band) and under 24 hour (lower band) postmortem interval groups. the postmortem interval (fig 1), and, except for $(\ell$ aminobutyric acid and valine, inversely related to the chronological age of the infant.

The increase in concentration with time after death was significant $(\mathrm{p}<0 \cdot 01)$ for six amino acids in the 13-24 hour postmortem interval group, 13 amino acids in the $25-48$ hour group, and 23 amino acids in the 49->96 hour group. In the combined over 24 hour groups $(n=62)$ the increase was significant $(\mathrm{p}<0 \cdot 002)$ for all 27 of the amino acids compared with those sampled less than 24 hours after death $(n=58)$.

The decrease in concentration with chronological age was significant $(\mathrm{p}<() \cdot(01)$ for only nine amino acids in the 27-52 weeks age group. Numbers in the $1-4$ weeks neonatal age group $(n=7)$ were too small for adequate comparison.

The mean concentration of 25 amino acids was higher in girls but the difference did not attain significance at the ().()1 level. In one amino acid $(\gamma-$ aminobutyric acid) the concentration was significantly higher $(p<0.002)$ in boys than girls.

Altogether $2433(82 \%)$ of all the $(2945)$ results were within the normal age related plasma reference range (3SD) for this hospital with $86 \%$ in the normal range in the combined under 24 hour postmortem interval group and $79 \%$ in the normal range in the over 24 hour group (fig 2). Hydroxyproline concentrations were unrecordable in $64 \%$ of cases in the under 24 hour group. Ethanolamine and $\gamma$-aminobutyric acid were excluded as age related plasma concentrations were not available.

Three cases had abnormal vitreous phenylalaline:tyrosine ratios and were probably examples of transient phenylalaninaemia occurring in early infancy, although in one case the ratio was particularly high $(36: 1)$. In a fourth case there was a decrease in the phenylalanine:tyrosine ratio $(1: 2 \cdot 8)$ entirely due to an increase in tyrosine concentration perhaps related to infantile tyrosinaemia.

\section{Discussion}

Postmortem changes occur less rapidly in vitreous humour than in other body fluids so that theoretically at least analysis of the vitreous humour could give useful information regarding a possible cause of cot death. ${ }^{6}$ Little is known about the progression of the biochemical changes after death so that in analysing such changes multiple variables should ideally be taken into account including for example feeding patterns, circadian rhythm, and latent infections, in addition to the parameters we have already considered.

The present study has indicated that samples should be obtained not more than 24 hours after 
death to give reliable results. The postmortem interval as already defined established fixed limits for the interval and avoided possible overestimation of the amino acid concentration relative to time after death.

Analysis of the results showed no evidence that a specific aminoacidopathy is implicated in the aetiology of cot death but has nevertheless improved our understanding of the biochemical background of these cases.

The baseline data for vitreous amino acid concentrations by age and postmortem interval in cot deaths may be of value to other investigators and is presented in a table that is available from the corresponding author.

We should like to thank Mr Finlay Sim and Mr Peter McAllister who undertook the amino acid analysis, Mr Jim Ito for help with computer analysis of the results, Mr Fergus Coleman for preparing the illustrations, and Mrs $\mathrm{R}$ Todd for typing the manuscript.

\section{References}

1 Arncil GC, Brookc H. Gibson AAM, Harvic A, McIntosh H,
Patrick WJA. National post-perinatal infant mortality and cot death study, Scotland 1981-82. Lancet 1985;i:740-3.

2 Erdei Z, Vass Z. Chromatographic investigation of free amino acids of the human vitrcous body. Acta Ophthalmol (Copenh) 1967:45:22-4.

${ }^{3}$ Durham DG, Dickinson JC. Hamilton PB. Ion-exchange chromatography of free amino acids in human intralocular fluids. Clin Chem 1971:17:285-9.

+ Froggatt P. Lynas MA. MacKenzie G. Epidemiology of sudden unexpected death in infants ('cot death') in Northern Ireland British Journal of Preventive and Social Medicine 1971:25: 119-34.

${ }^{5}$ Beckwith JB. Observation on the pathological anatomy of the sudden infant death syndrome. In: Bergman AB, Beckwith JB, Ray CG, eds. SIDS: proceedings of the second international conference on causes of sudden death in infants. Seattle, University of Washington Press, 1970:83-107.

- Blumenfeld TA. Mantell CH, Catherman RL, Blanc WA. Postmortem vitreous humor chemistry in sudden infant death syndrome and in other causes of death in childhood. Am J Clin Pathol 1979:71:219-23.

Correspondence and requests for reprints to Dr WJA Patrick. Royal Hospital for Sick Children, Yorkhill, Glasgow G3 8SJ.

Accepted 1 February 1988
SUMmary We describe seven children with subcutaneous rheumatoid nodules who had no clinical evidence of rheumatoid arthritis. Only one girl was seropositive for antinuclear factors and had a slightly raised erythrocyte sedimentation rate. Clinical aspects, risks for developing rheumatoid arthritis, and treatment of this entity are discussed.

Subcutaneous tumours that histologically resemble rheumatoid nodules are rarely encountered in healthy children - that is, those without evidence of arthritis. Unfamiliarity with these lesions often gives rise to uncertainty concerning the subsequentpossible-occurrence of rheumatoid arthritis and the treatment of the frequently appearing recurrences. In this article we review our own data and compare it with results found in the literature.

\section{Patients and methods}

We reviewed the notes of seven children who had been referred to the department of paediatric surgery at the St Radboud University Hospital in
Nijmegen between January 1970 and October 1986 with subcutaneous lesions, which were histologically undistinguishable from the nodules found in adults with rheumatoid arthritis. All children were invited to take part in a follow up examination. The response rate was $100 \%$; the period of follow up varied from 6 months to 17 years (mean 6.3 years).

\section{Results}

At the first presentation to hospital all children were found to be healthy and none of them had a history of joint disease. Clinical details of the children are shown in the table. The group consisted of two boys and five girls, and their mean age was 5.5 years (ranging from 6 months to 10 years). A solitary nodule had been diagnosed in four children, one child had had two nodules, and two had presented with multiple nodules. Most lesions had been found in the extensor side of extremities: four pretibial, one prepatellar, one on the dorsum of the foot, and one on the extensor side of a finger. In two children the lesions had been situated on the scalp. The size of the nodules ranged from $2 \mathrm{~mm}$ to $4 \mathrm{~cm}$. Ten of the subcutaneous nodules had been excised and histo- 